\title{
Polyaniline Enhanced Supercapacitance of Cobalt Hydroxide Nanowires/Carbon Nanotube Containing Polymer Sponge Layered Composite
}

\author{
SYED Hashim Shah ${ }^{\mathrm{a}}$, MUHAMMAD Imran Khan ${ }^{\mathrm{b}^{*}}, \mathrm{RIDA}^{\mathrm{S}}$ Sarfraz ${ }^{\mathrm{c}}$, \\ ROZEEN Nazir ${ }^{d}$, ASHRAF Ali ${ }^{\mathrm{e}}$
}

\author{
Faculty of Materials and Chemical Engineering, Ghulam Ishaq Khan Institute of Engineering \\ Sciences and Technology, Topi, Swabi, Pakistan \\ ahashim@giki.edu.pk, bimrankhan@giki.edu.pk, cridaskhan29@yahoo.com, \\ drozeen.nazir@gmail.com, edrashraf@giki.edu.pk
}

Keywords: Supercapacitor, Polyaniline, Polymer Foam, Energy Storage, Electrochemical Composites.

\begin{abstract}
Transition metal oxide nanostructures and conducting polymers like polyaniline have specific capacitance, an order of magnitude higher than those of carbon based nano-materials. In the present study, nano-flowers of cobalt hydroxide, polyaniline and carbon nanotubes were combined on a conventional polymeric sponge to develop by using facile wet chemical techniques. High surface area of cobalt hydroxide nano-flowers when combined with polyaniline showed enhanced capacitance values and stability. The carbon nanotubes enhanced the conductivity of the composite while the double porous structure of polyurethane sponge enhances the electrolyte flow, surface area, and reduces current density which leads to good reversibility and greater capacitance. Samples were characterized by cyclic voltammetry technique against $\mathrm{Ag} / \mathrm{AgCl}$ reference electrode in three electrode setup.
\end{abstract}

\section{Introduction}

Super-capacitors are an attractive class of energy storage devices which have potential to replace batteries. They have relatively higher charge and discharge rate, higher cyclic life and high power density [1,2]. There are two mechanisms involved in energy storage in them i.e. electric double layer effect and pseudo-capacitive effect [1,3]. Graphene, carbon nanotubes, and various porous carbons store energy by adsorption of ions on their high surface area pores, which create double layers of ions. The electric field in this extremely thin double layers can be treated as dielectric layer of single molecular thickness which stores the charge as shown by the Eq. 1 [4].

$$
C=\frac{\varepsilon A}{d}
$$

Where $\mathrm{C}$ is the capacitance, $\varepsilon$ is dielectric constant and $\mathrm{d}$ is the thickness of double layer. The very small distance between ions give rise to very high capacitances which can be further enhanced by increasing surface areas [4]. Whereas the pseudo-capacitive materials undergo quick faradaic redox reactions whose cyclic voltammograms looks similar to that of capacitors. Many transition metal oxides show this effect. Ruthenium Oxide was the first oxide that was discovered exhibiting this phenomenon [5].

Currently the best carbon EDLCs have specific capacitances of $150 \mathrm{~F} \cdot \mathrm{g}^{-1}$ [5]. Carbon alone shows comparatively lower capacitance. Carbon nanotubes and graphene are also promising materials. Microwave exfoliated graphene shows capacitance of $191 \mathrm{~F} / \mathrm{g}$ in $\mathrm{KOH}$ [6]. Many groups have formulated composites of oxides, conducting polymers, and carbon based materials to meet the required applications such as; manganese dioxide/activated carbon based super-capacitor was made by Yuan et al. using lithium hydroxide as electrolyte and obtained $62.4 \mathrm{~F} / \mathrm{g}$ [7], Sen et al. combined manganese dioxide nanorods with polyaniline and PEDOT to obtain capacitance of $221 \mathrm{~F} / \mathrm{g}$ and $315 \mathrm{~F} / \mathrm{g}$ respectively [8], Zhu et al. combined polyaniline nano fibers with graphite oxide via interfacial polymerization and obtained energy density of $7.1 \mathrm{Wh} / \mathrm{kg}$ and a power density 
of $80 \mathrm{~W} / \mathrm{g}$ [9]. In another study, the addition of redox active electrolytes was shown to increase the capacitance of polyaniline coated curved graphene upto $96 \%$ compared to without redox active contributors in electrolyte [10].

Cobalt Hydroxide is an important class of material used in energy storage because it has very high theoretical capacitance of $3458 \mathrm{~F} / \mathrm{g}$. [11]. It undergoes phase transformation which leads to constant potential during galvanostatic charge discharge. When nanostructured, its capacity can be highly enhanced [5]. The only problem with these high capacity oxides is their extremely low conductivity. This can be enhanced by making composites with conductive materials. In this regard Carbon nanotubes have emerged as a potential material which are highly conductive, stable and non-reactive. Multi-walled (MW) Carbon nanotubes also offer excellent conductivity due to absence of electrons scattering [2]. Cheng et al. designed a composite of graphene intercalated with cnts and cobalt hydroxide nano-sheets in between. The asymmetric super-capacitor with an ionic liquid electrolyte give very high energy density and power density of $172 \mathrm{Wh} / \mathrm{kg}$ and $198 \mathrm{~kW} / \mathrm{kg}$ [11]. Jagadale et al. evaluated performance of cobalt hydroxide thin films and reported a maximum specific capacitance of $44 \mathrm{~F} / \mathrm{g}$ [12]. In another work, electrochemical deposited hybrid nanocomposite of $\mathrm{Co}(\mathrm{OH})_{2}$ and polyaniline was prepared with a reported capacitance of $215 \mathrm{~F} / \mathrm{g}$ [13] .

More recently, conductive polymers have also been shown to possess pseudo-capacitive properties and have been used in energy storage devices[10,14-16]. Polyaniline is stable, highly conductive and easy to polymerize. It raised a considerable amount of interest in the research arena in energy storage and sensors [16-23].

Moussa et al. have combined carbon based materials with polyaniline on polymer sponge and have achieved very high capacitances [16]. The sponge acts as a highly porous and flexible replacement of conventional nickel foam. Similarly, Chen et al. used combined $\mathrm{MnO}_{2}$ with carbon nanotubes on a polymer sponge and achieved a capacitance of $1230 \mathrm{~F} / \mathrm{g}$. They also reported high cyclic life and high specific power of $63 \mathrm{~kW} / \mathrm{kg}$ [24]. However, a quaternary composite has not yet been tried as per our knowledge. In this work, to circumvent problems posed by oxides and overcome deficiencies discussed above, we investigate a quaternary composite of MW carbon nanotubes, Polyaniline and in-situ grown cobalt hydroxide nanowires on a polymer sponge as a potential electrode material for super capacitors.

\section{Experimental}

Materials. Commercial polyurethane sponge, acetone and propanol were used for cleaning of sponge. For synthesis of electrode materials, multi-walled carbon nanotubes, sodium dodecyl sulphate, cobalt chloride, urea, aniline, $\mathrm{HCl}$ and ammonium persulfate were used.

After 24 hours, due to Vander wall attractions, the MWCNTs were deposited on the on the foam strands. The coated foam was dried, gently washed in distilled water to remove the surfactant and then dried again. The resulting foam was highly conductive. The coating is shown in the Fig. 1.

CNT Coating on the PU Sponge. Before coating, the sponge samples were cut into small flat pieces by means of a punch. They were washed with acetone, iso-propyl alcohol, and distilled water to remove any organic and inorganic residues. They were dried at $333 \mathrm{~K}$ in an oven for 24 hours. After weighing the sponges carefully, they were placed in the MWCNT Suspension. Care was taken to squeeze the sponge to take out any trapped air bubble that might hinder the coating. To make the suspension, $100 \mathrm{mg}$ multiwall CNT and $750 \mathrm{mg}$ of sodium dodecyl sulfate were weighed and mixed in $50 \mathrm{ml}$ of water. Afterwards the solution was sonicated for around 1 hour to prepare a uniform dispersion of CNT in water. 



Fig. 1. (A) Polyurethane foam. (B) carbon nanotube coated foam. (C)Polyaniline-CNT coated foam. (D) Magnified image of polyaniline coating showing fibrous structure. (E) Cobalt hydroxide-polaniline-CNT foam. (F) Magnified image of cobalt hydroxide coating showing globular structure. (G) Nano-flower like morphology of cobalt hydroxide. (H) Higher magnification image of cobalt hydroxide nano-wire flowers. The structure shows high porosity and long wires emanating from single point.

Polyaniline Coating. Firstly, $40 \mathrm{mmol}$ aniline was weighed on a digital weighing balance. Once the aniline was weighed, it was added in $20 \mathrm{ml} 1 \mathrm{M} \mathrm{HCl}$. Once the aniline solution was prepared, sponges were dipped into it, and gas bubbles were squeezed out with the help of a glass rod. This whole procedure was made to take place at $313 \mathrm{~K}$ and then polymerized with acidic ammonium persulfate solution which was prepared by adding $4 \mathrm{mmol}$ ammonium per sulphate in to $20 \mathrm{ml} 1 \mathrm{M}$ $\mathrm{HCl}$ at $278 \mathrm{~K}$. After mixing, the solution was slowly turned greenish in color and a very thin layer of green polyaniline was deposited on the sponge.

Cobalt Hydroxide Nano-wire growth. Cobalt Hydroxide nanowires were grown by chemical bath deposition process. For preparation of precursor solution $6.4 \mathrm{~g}$ cobalt chloride was weighed and mixed with $100 \mathrm{~g}$ urea in $500 \mathrm{ml}$ distilled water taken in a beaker. The sponge was dipped into the solution and kept at $330 \mathrm{~K}$ for 24 hours with beaker enclosed. As a result of this mixing, following reaction takes place slowly.

$$
\left[\mathrm{Co}\left(\mathrm{H}_{2} \mathrm{O}\right)_{6}\right]^{2+}(\mathrm{aq})+2 \mathrm{OH}-(\mathrm{aq}) \rightarrow\left[\mathrm{Co}\left(\mathrm{H}_{2} \mathrm{O}\right)_{4}(\mathrm{OH})_{2}(\mathrm{~S})+2 \mathrm{H}_{2} \mathrm{O}\right.
$$

The slow reaction deposits clusters of porcupine like nano-wires as shown in electron microscopy images shown in Fig.1.

Electrochemical Characterization methods. The fabricated electrodes were tested in a three electrode setting with $\mathrm{Ag} / \mathrm{AgCl}$ Reference Electrode and graphite counter electrode in $2 \mathrm{M} \mathrm{KOH}$ electrolyte in water. Working electrode was prepared by attaching foam on highly compacted graphite piece via small amount of conductive silver paste. Prior to testing, the working electrode was kept in the electrolyte for proper soaking and stabilization. The capacity and cyclic life was estimated by cyclic voltammetry technique. Specific capacitance was calculated by using the formula

$$
C=\frac{1}{2 m \Delta V} \int_{V_{\text {initial }}}^{V_{\text {final }}} \frac{I}{d V / d t} d V
$$

Where $\mathrm{m}$ is the mass of electrode, $\Delta \mathrm{V}$ is the voltage range in which the cyclic voltammetry is conducted and $\mathrm{dV} / \mathrm{dt}$ is the scan rate.

Energy density was also calculated using the formula below

$$
E=\frac{1}{2} C_{\text {specific }} V^{2}
$$




\section{Results and Discussion}

The CNT was deposited on the foam in the form of rough agglomerates as shown in the Fig 1. The coating as was quite adherent owing to entanglement of carbon nanotubes and Van der Waal interaction between themselves and the PU foam. The continuous network makes the foam conductive. Raman spectra (Fig. 3) of CNT coated sponge showed two peaks. The peak at $1327 \mathrm{~cm}^{-}$ ${ }^{1}$ can be identified with D band which should theoretically be present at $1350 \mathrm{~cm}^{-1}$. However due to functionalization or diameter change, this band can be slightly shifted. Similarly, $G$ band of the CNT's is detected at $1572 \mathrm{~cm}^{-1}$, however this band is theoretically present at $1582 \mathrm{~cm}^{-1}$. The subsequent polyaniline coating completely covers the CNT coated foam and changes the color foam to dark green. Following the chemical bath deposition of the cobalt hydroxide nanowires the foams become pink and very rough as seen in the visual inspection and electron micrographs (Fig. 1). The adopted chemical bath deposition method is a slow procedure and allows for growth of pristine cobalt hydroxide nanowire clusters directly on the substrate due to heterogeneous nucleation. This in-situ growth enables higher adhesion of wires on to the substrate leading allowing a binder free electrode.

Electrochemical characterization showed significant enhancement in capacitance values with addition of cobalt hydroxide and polyaniline as compared to simple CNT coated polyurethane sponge. Compared to CNT sponge, which shows a specific capacitance of $355 \mathrm{~F} / \mathrm{g}$, CNT-cobalt hydroxide sponge and CNT-polyaniline-cobalt hydroxide showed a specific capacitance of $620 \mathrm{~F} / \mathrm{g}$ and $920 \mathrm{~F} / \mathrm{g}$ which corresponds to a raise of $74 \%$ and $160 \%$, respectively. The raise in specific capacitance value is near double with polyaniline addition and can be attributed to pseudo capacitance contribution by polyaniline. In case of polyaniline loaded sponge, the immediate fall of capacitance upon charge and discharge can be contributed to the de-doping of polyaniline into nonconductive emeraldine base due to $\mathrm{KOH}$ electrolyte. The insulation state has high resistance to charge flow therefore low capacitance. However, due to charge and discharge, the polyaniline expands which leads to number of spaces between wires to increase thus allowing more electrolyte flow, hence improving the capacitance overall. The cyclic voltammograms of the composites in Fig.2 show significant differences from each other in terms of shape position of redox peaks. The CNT foam shows a very sleek CV curve typical of EDLCs while the cobalt hydroxide containing electrodes show redox peaks. The presence of polyaniline changed the discharge dip from $-30 \mathrm{mV}$ to $91 \mathrm{mV}$.
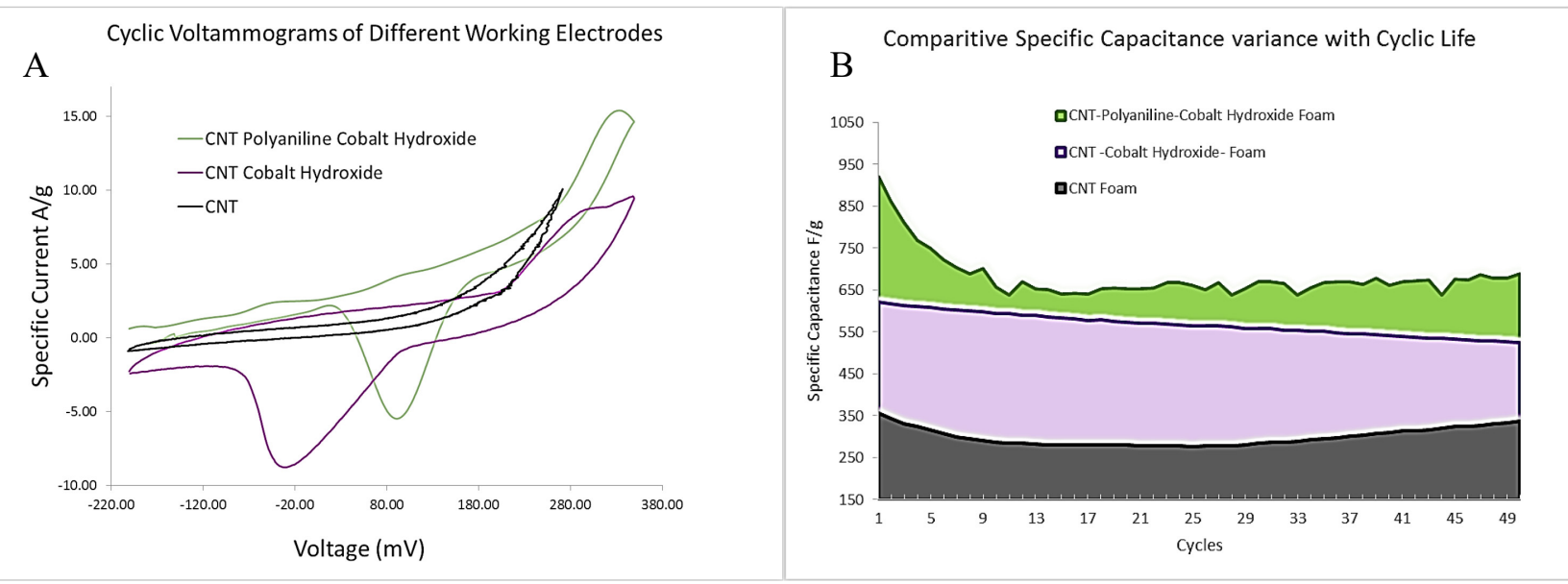

Fig. 2. (A) The cyclic voltammograms of CNT (black), CNT-cobalt hydroxide (pink), and CNT-polyanilinecobalt hydroxide (green) coated sponges. The CNT coated sponge shows very small currents and charge storage capacity. The cobalt hydroxide containing samples show Redox Peaks typical of pseudo capacitive behavior. (B) The capacity vs cyclic life curve for the 3 electrodes materials. There is constant fall in the capacity of the CNT-CO Sample as compared to rapid-then-persistent capacitance value of CNT-PA-CO. 
During the cobalt hydroxide coating procedure, we also investigated the cobalt hydroxide deposition rate and found a linear dependence of mass gain in thin film against the time. But very thick coatings are undesirable as they limit the amount of active material exposed for redox reactions and are very prone to cracking in case of mechanical shock or electrochemical stresses. Fig. 4 shows cracks in cobalt hydroxide layer due to mechanical stresses produced due to compression of foam.

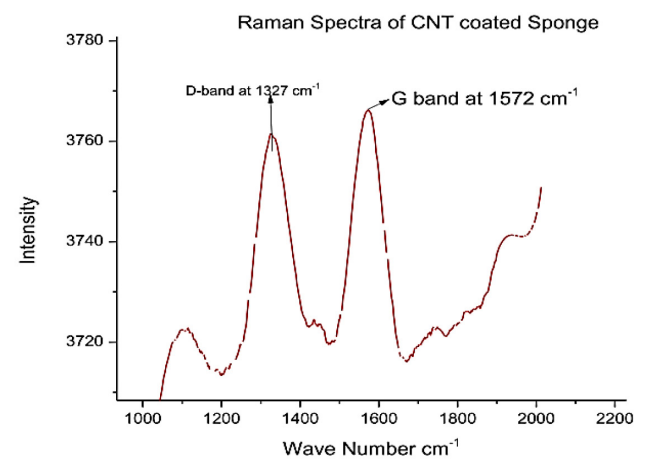

Fig. 3. Raman spectrum of CNT coated polyurethane foam showing the typical CNT peaks.

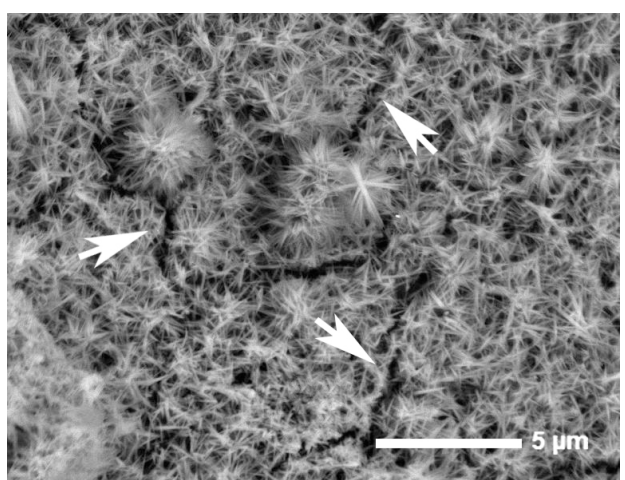

Fig. 4. Cracks shown in thick coatings of cobalt hydroxide.

\section{Conclusions}

Polyaniline addition enhanced the specific capacitance by $48 \%$ as compared to CNT-CO foam. The rapid fall of capacitance in case of polyaniline addition can be attributed to the de-doping and reducing in conductivity of polyaniline in presence of concentrated $\mathrm{KOH}$. This problem may be overcome by use of acidic or neutral salt electrolytes. The use of polymer foam as a template for coating is suitable because of wide macro pores, flexibility and durability. The foam interior is protected from mechanical abrasions therefore the active material is protected in case of mechanical abuse. It is also cheaper and much lighter than conventional metallic foam. The in-situ growth allows for good adherence of cobalt hydroxide nanowires which also reduces delamination in binder free electrode.

\section{References}

[1] A. González, E. Goikolea, J. A. Barrena, R. Mysyk, Review on supercapacitors: technologies and materials, Renew. Sustain. Ener. Rev., 58 (2016) 1189-1206.

[2] F. Wang, S. Xiao, Y. Hou, C. Hu, L. Liu, Y. Wu, Electrode materials for aqueous asymmetric supercapacitors, RSC Adv., 3 (2013) 13059-13084

[3] J. Yan, E. Khoo, A. Sumboja, P. S. Lee, Facile coating of manganese oxide on tin oxide nanowires with high-performance capacitive behavior., ACS Nano, 4 (2010) 4247-55.

[4] F. Béguin, V. Presser, A. Balducci, E. Frackowiak, Carbons and electrolytes for advanced supercapacitors, Adv. Mater., 26 (2014) 2219-2251.

[5] V. Augustyn, P. Simon, B. Dunn, Pseudocapacitive oxide materials for high-rate electrochemical energy storage, Ener. Environ. Sci., 7 (2014) 1597-1614.

[6] Y. Zhu, S. Murali, M. D. Stoller, A. Velamakanni, R. D. Piner, R. S. Ruoff, Microwave assisted exfoliation and reduction of graphite oxide for ultracapacitors, Carbon, 48 (2010) 2118-2122.

[7] A. Yuan, Q. Zhang, A novel hybrid manganese dioxide/activated carbon supercapacitor using lithium hydroxide electrolyte, Carbon, 26 (2006) 321-329.

[8] P. Sen, A. De, A. D. Chowdhury, S. Bandyopadhyay, N. Agnihotri, M. Mukherjee, Conducting polymer based manganese dioxide nanocomposite as supercapacitor, Electrochim. Acta, 108 (2013) 265-273. 
[9] J. Zhu et al., Interfacial polymerized polyaniline/graphite oxide nanocomposites toward electrochemical energy storage, Carbon, 12 (2012) 121-128.

[10] W. Chen, R. B. Rakhi, H. N. Alshareef, Capacitance enhancement of polyaniline coated curved-graphene supercapacitors in a redox-active electrolyte., Nanoscale, 5 (2013) 4134-8.

[11] Q. Cheng, J. Tang, N. Shinya, L.-C. Qin, Co (OH) 2 nanosheet-decorated graphene-CNT composite for supercapacitors of high energy density, Sci. Techno. Advan. Mater., 15 (2014) 014206.

[12] A. Jagadale, V. Kumbhar, D. Dhawale, C. Lokhande, Performance evaluation of symmetric supercapacitor based on cobalt hydroxide $[\mathrm{Co}(\mathrm{OH}) 2]$ thin film electrodes, Electrochim. Acta, 98 (2013) 32-38.

[13] J. H. Shendkar et al., Polyaniline-cobalt hydroxide hybrid nanostructures and their supercapacitor studies, Mater. Chem. Phy., 180 (2016) 226-236.

[14] L. Zhang, L. Chen, B. Qi, G. Yang, J. Gong, Synthesis of vertical aligned TiO 2@ polyaniline core-shell nanorods for high-performance supercapacitors, RSC Advances 5 (2015) 1680-1683.

[15] L. Chen et al., Synthesis and electrochemical performance of polyaniline-MnO 2 nanowire composites for supercapacitors, J. Phy. Chem. Soli., 74 (2013) 360-365.

[16] M. Moussa et al., High-performance supercapacitors using graphene/polyaniline composites deposited on kitchen sponge, Nanotechnol., 26 (2015) 075702.

[17] A. Abdolahi, E. Hamzah, Z. Ibrahim, S. Hashim, Synthesis of uniform polyaniline nanofibers through interfacial polymerization, Mater., 5 (2012) 1487-1494.

[18] C.-L. Zhu, S.-W. Chou, S.-F. He, W.-N. Liao, C.-C. Chen, Synthesis of core/shell metal oxide/polyaniline nanocomposites and hollow polyaniline capsules, Nanotechnol., 18 (2007) 275604.

[19] N. Muthukumar, G. Thilagavathi, T. Kannaian, Polyaniline-coated polyurethane foam for pressure sensor applications, Hi. Perform. Poly., 28 (2016) 368-375.

[20] F. Chen, C. Yang, Study on Preparation of Conducting Polyaniline/Polyurethane Foam and Its Use in Silver Ion Recovery, Polymer. Sci., 25 (2011) 112-117.

[21] A. Dey, S. De, A. De, S. De, Characterization and dielectric properties of polyaniline-TiO2 nanocomposites, Nanotechnol., 15 (2004) 1277-1285.

[22] J. Huang, S. Virji, B. H. Weiller, R. B. Kaner, Polyaniline nanofibers: facile synthesis and chemical sensors., J. Am. Chem. Soc., 125 (2003) 314-5.

[23] S. M. Pethe, S. B. Kondawar, Optical and electrical properties of conducting polyaniline nanofibers synthesized by interfacial and rapid mixing polymerization, Advan. Mater. Lett., 5 (2014) 728-733.

[24] W. Chen, R. Rakhi, L. Hu, X. Xie, Y. Cui, H. N. Alshareef, High-performance nanostructured supercapacitors on a sponge, Nano Lett., 11 (2011) 5165-5172. 